\title{
Tecnologías de energías renovables (TER) desde el compromiso público
}

\section{Renewable Energy Technologies (RET) from Public Engagement}

\author{
Adriana-Patricia Gallego-Torres ${ }^{1}$ \\ Vladimir-Alfonso Ballesteros-Ballesteros ${ }^{2}$
}

Recibido: marzo de 2021

Aceptado: agosto de 2021

Para citar este artículo: Gallego-Torres, A. P. y Ballesteros-Ballesteros, V. A. (2021). Tecnologías de energías renovables (TER) desde el compromiso público. Revista Científica, 42(3), 368-377.

https://doi.org/10.14483/23448350.18586

\section{Resumen}

Este trabajo presenta una revisión extensa acerca de la importancia de las posturas ciudadanas en la implementación de las tecnologías de energías renovables (TER). En las últimas décadas la educación ha intentado comprender desde el compromiso público la aceptación social de las tecnologías de energías renovables y la participación de las comunidades en los procesos de toma de decisiones relacionados con la transición hacia la implementación de energías limpias. En este sentido, el presente trabajo hace una reflexión teórica sobre el compromiso público, la aceptación e implementación de modelos de tecnologías en energías renovables y la participación ciudadana.

Palabras clave: compromiso público; educación; energías renovables; tecnologías.

\begin{abstract}
This work presents an extensive review about the importance of citizen positions in the implementation of renewable energy technologies (RET). In the last decades, education has tried to understand, from the perspective of public commitment, the social acceptance of renewable energy technologies and the participation of communities in decision-making processes related to the transition towards the implementation of clean energies. In this sense, this study makes a theoretical reflection on public commitment, the acceptance and implementation of renewable energy technology models, and citizen participation.
\end{abstract}

Keywords: education; public engagement; renewable energies; technologies.

1. Ph. D. Universidad Distrital Francisco José de Caldas, Bogotá D.C., Colombia. adpgallegto@udistrital.edu.co

2. Ph. D. (c) Fundación Universitaria Los Libertadores, Bogotá D.C., Colombia. vladimir.ballesteros@libertadores.edu.co 


\section{Introducción}

Este trabajo se desarrolla a partir de tres dimensiones fundamentales, en primer lugar, la noción de simetría como punto de partida para la evaluación de la aceptación social y las relaciones de la ciencia, la sociedad y el medio ambiente (Lyall y Tait, 2017; Rip, 2018; Kreimer y Vessuri, 2018; Berg y Lidskog, 2018), y la transición de un modelo de déficit, con énfasis en comunicación en doble vía en forma de diálogo, al compromiso público ascendente (Wilsdon y Willis, 2004; MacFarlane, 2018), con el objetivo de restaurar la confianza y aceptación pública y construir una mayor capacidad reflexiva en la implementación de los desarrollos de la ciencia y las tecnologías energéticas (Wilsdon, Wynne y Stilgoe, 2005; Martin, 2017; Mor y Reich, 2018; Hsieh, Vaickus y Remick, 2018).

En segundo lugar, el modelo TER y la participación de las energías renovables en las agendas políticas de los gobiernos, lo que ha resaltado la necesidad de desarrollar procesos de implementación y transición social comunitaria (Rees y Wackernagel, 1996; Brown y Ulgiati, 1999, Batel, 2020). Esto supone reconocer que las energías renovables constituyen una opción sostenible para el suministro energético en diferentes zonas geográficas del planeta, pero también que es necesario realizar un proceso de compromiso público de los ciudadanos que permita la utilización y el normal desarrollo (Wüstenhagen, Wolsink, y Bürer, 2007; Li y Zheng, 2016; Furlan y Mortarino, 2018, Salamanca-Céspedes, 2019). Sin embargo, la acción concertada entre distintos actores para promover el apoyo a las políticas publicas para la incorporación de las energías renovables sigue provocando controversia en distintos países, incluso en aquellos con capacidad política y económica para transformar prácticas culturales (Lilliestam y Hanger, 2016; Kropp, 2018).

En tercer lugar, la evolución de la aceptación publica del compromiso público desde las distintas formas de participación de la ciudadana que involucra al público en la deliberación de nuevas políticas y prácticas energéticas (Dinica, 2018; Neshkova y Guo, 2018). De este modo, la participación ciudadana representa un mecanismo, cada vez más importante, que contribuye a un renacer político a partir de prácticas innovadoras (Speer y Han, 2018; Alsina y Martí, 2018); esta participación proporciona, por lo menos en teoría, un espacio de empoderamiento ciudadano y un lugar privilegiado para contribuir a la resolución de problemas ambientales y sociales que los involucra (Gustafsson, Ivner y Palm, 2015; Cardullo y Kitchin, 2018).

\section{Antecedentes: la noción de simetría como punto de partida en la aceptación social}

La noción de simetría definida por Walker (1995) es el punto de partida para la construcción de una nueva perspectiva sobre la educación en energías renovables desde el campo de la evaluación constructiva de la tecnología y que tiene por objeto movilizar ideas sobre la dinámica coevolutiva de la ciencia, la tecnología y la sociedad para anticipar y evaluar las tecnologías, en lugar de ocuparse predominantemente de la aceptación y evaluación de los efectos sociales de una tecnología cuasi regulada (Schot y Rip, 1997). Desde este punto de vista, la noción de simetría, es decir, que la participación pública abarca no solo las reacciones del público a las propuestas tecnológicas, sino también las acciones de los agentes que participan en la promoción del desarrollo tecnológico y en la interacción con el público de diversas maneras.

\section{Modelo TER como punto de inflexión}

Este modelo, en primera instancia, busca conceptualizar los elementos y procesos que intervienen en la configuración de las interacciones que tienen lugar entre los promotores de la tecnología y el público local en relación con las propuestas de desarrollo de las TER (Batel, 2020). Su objetivo es ser descriptivo y explicativo más que prescriptivo o normativo. No sugiere cómo deberían tener lugar 
las interacciones ni cómo debería practicarse el compromiso. Tampoco intenta ser simplista y predictivo, ni sobre cómo se desarrollarán las respuestas en una situación dada o sobre cómo pueden resultar las decisiones finales acerca de la aprobación de los proyectos. En segunda instancia, no intenta ser totalmente inclusivo, abarcando todos los elementos, procesos o interconexiones causales que puedan ser relevantes para el desarrollo de un proyecto de TER. Como modelo, es inevitablemente abstracto hasta cierto punto, y selectivo en lo que destaca y en lo que minimiza. Este modelo también trata de captar las expectativas que entraña la forma en que las personas y las organizaciones tratan de relacionarse, o no, con los demás, la naturaleza dinámica de esas expectativas y respuestas a lo largo del tiempo y la contextualidad de la participación, moldeada tanto por los amplios paisajes políticos y económicos como por las características de los lugares y las comunidades locales (Ballesteros-Ballesteros y Gallego-Torres, 2019; Ortega y Gil, 2019).

Las respuestas de los distintos públicos a proyectos de inversión y desarrollo relacionados con TER no se desarrollan en el vacío o en lo abstracto, sino en interacción con otros que tienen un interés en un desarrollo (particularmente aquellos que lo defienden y lo promueven). Por lo tanto, en lugar de identificar personas predispuestas y en condición de oponerse o apoyar desarrollos particulares, se deben entender las respuestas locales como emergentes, negociadas y cambiantes (Futrell, 2003) en relación con una variedad de factores asociados al contexto local o regional (Franco, Reina y Riveros, 2019).

Por ejemplo, las explicaciones de las iniciativas NIMBY para la oposición a las propuestas de desarrollo han sido criticadas por centrar la atención exclusivamente en el público, a menudo de manera peyorativa (Wolsink, 2000; Petrova, 2016). Así, la oposición se explica por el hecho de señalar a las personas por preocuparse solo por sus propios intereses y por el valor de sus propiedades (Bidwell, 2016a). No se presta atención a lo que los desarrolladores y promotores de las TER están haciendo y diciendo, ni a cómo se estructuran y promulgan los procesos de toma de decisiones (Burningham, Barnett y Walker, 2015).

\section{Materiales y métodos}

El presente artículo es el fruto de una revisión sistemática y exploratoria a partir del planteamiento de categorías teóricas relacionadas con la educación en energías renovables, el compromiso público y las tecnologías de energías renovables (TER). La bibliografía seleccionada fue recuperada de bases de datos especializadas. En total se analizaron cerca de cien artículos que incluían las categorías preestablecidas y que abarcaban un periodo de 15 años, y se clasificaron según sus principales desarrollos para responder las preguntas motivadoras de análisis: ¿cómo ha evolucionado el concepto de las tecnologías de energías renovables y qué enfoques emergentes han aparecido? y ¿cuáles son las necesidades de investigación futura en este campo?

\section{Resultados y discusión}

La literatura revisada define las tecnologías de energías renovables TER a partir de la publicación de Walker (1995), quien vaticinaba la necesidad de tener en cuenta las actitudes de los ciudadanos hacia los proyectos de las energías renovables y el conflicto que podía aparecer particularmente dentro de los procesos de aprobación e implementación. Este campo de investigación lo retoman en 2007 Rolf Wüstenhagen, Maarten Wolsink y Mary Jean Bürer definiendo esta problemática como la aceptación social de las tecnologías de energía renovable, conceptualizándola a partir de tres dimensiones: la aceptación sociopolítica, la aceptación comunitaria y la aceptación del mercado. También surgieron investigaciones relacionadas con la opinión publica de los ciudadanos (Firestone y Kempton, 2007). A partir de estos trabajos se creó toda una línea de investigación relacionada con las actitudes energéticas, la comprensión 
publica de las energías renovables, la aceptación social y la sistematización de investigaciones que permitieron superar la explicación de NIMBY (no en mi patio trasero, Not In My Back Yard por su sigla en inglés) y una orientación para lo que hoy en día se conoce la aceptación social de TER (Betel, 2020).

En síntesis, el problema que surge hace referencia a la necesidad de involucrar al público en general a la hora de diseñar, gestionar e implementar proyectos de innovación y desarrollo tecnológico para las energías renovables, de tal manera que las representaciones sociales que los ciudadanos han elaborado al respecto de las principales energías renovables (eólica, fotovoltaica y biomasa) no sean un limitante a la hora de su implementación.

Desde esta perspectiva, se presenta el modelo TER (Figura 1), basado en la propuesta de Walker (1995). En el centro de este modelo, y de manera intencional, se encuentran las interacciones entre los "protagonistas de las TER en redes" y los "agentes públicos en el lugar". Estos agentes públicos que aparecen situados en lugares, comprenden lugares y comunidades particulares que son el foco de las propuestas de desarrollo de las TER, dando respuestas que también están situadas en contextos espaciales y culturales particulares. La denominación "agentes públicos" pretende abarcar tanto a los individuos como a los colectivos de los que pueden formar parte o llegar a formar parte. Se puede identificar que los "protagonistas de las TER" comprenden una amplia categoría de personas en organizaciones con funciones de apoyo o implementación de desarrollos de TER, incluyendo desarrolladores, consultores, compañías de relaciones públicas y marketing, asociaciones comerciales, financieros y fabricantes de tecnología. Estos actores se caracterizan por operar en redes, a través de las cuales se intercambia información, conocimientos especializados y experiencias, a veces en competencia o a veces en colaboración e interés mutuo (Walker (1995); Walker et al., 2010).

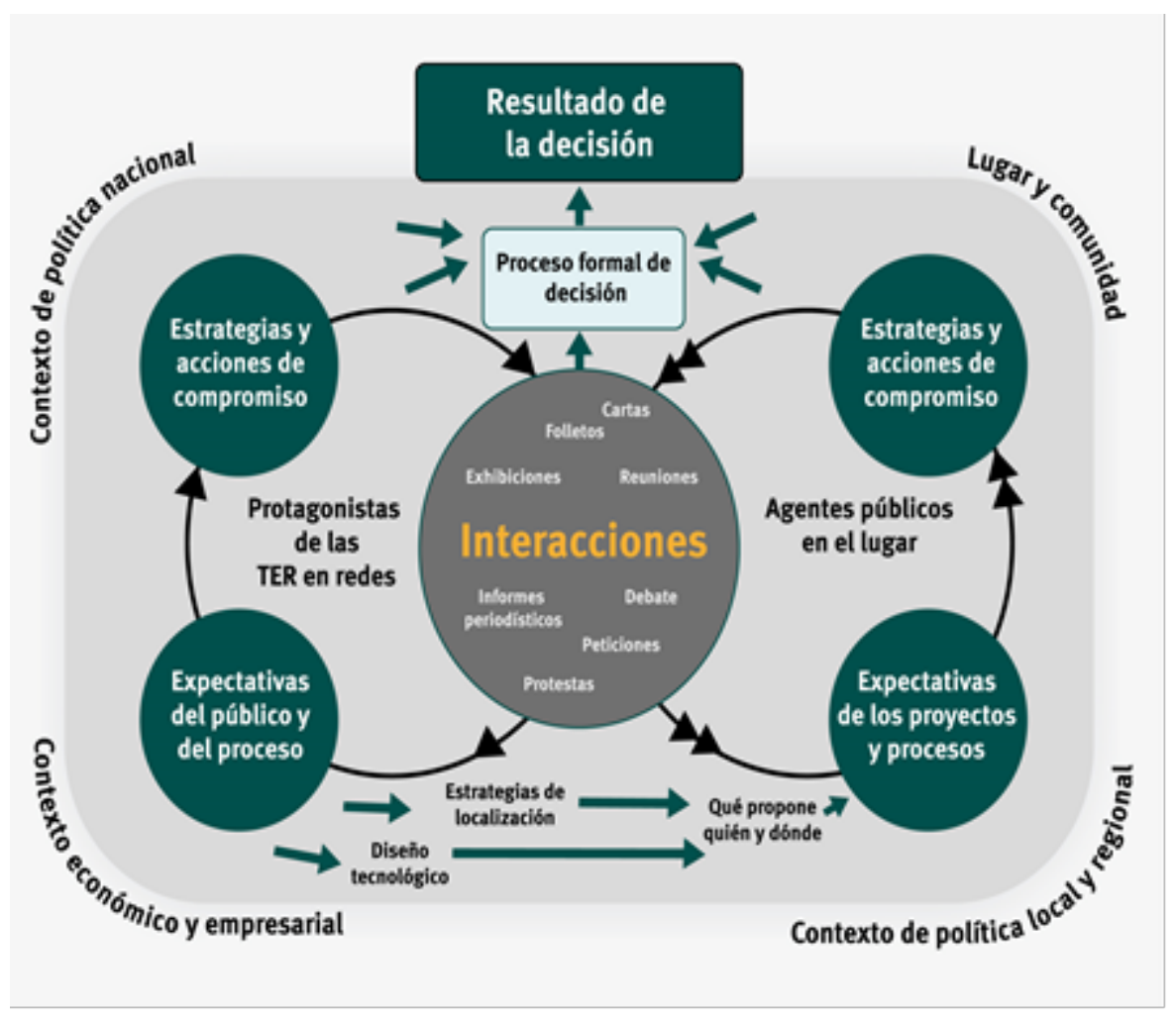

Figura 1: Adaptación del Modelo de Walker. 
La distinción generalizada entre "actores públicos en el lugar" y "protagonistas de las TER en redes", propuesta por los autores, se puede instalar como válida, aunque no irrefutable. Es evidente que los actores públicos pueden tener redes que se extienden más allá de sus lugares y comunidades específicas; por ejemplo, a través de la participación de grupos regionales o nacionales en disputas locales, o de la creación de conexiones estratégicas entre grupos locales con un interés común pero situados en lugares diferentes. Pero tales redes extendidas todavía tienen sentido y se utilizan para lograr la agencia y el impacto en los procesos locales basados en el lugar. Los actores de las TER pueden tener conexiones o asociaciones con lugares particulares, pero sin duda son más "libres". Sus redes e intereses no están limitados en el mismo grado por el lugar. Más bien, están estructurados funcional y profesionalmente con redes que se extienden más allá de las fronteras locales o regionales y, a menudo, nacionales dentro de empresas más grandes y cadenas de suministro globalizadas (Walker y Cass, 2007).

Entre estas dos categorías de actores, se sitúa una circunferencia turbia de interacciones: un espacio donde los actores de las TER y los actores públicos se comunican, intercambian información y opiniones, se conocen y se relacionan entre sí; estas interacciones son, potencialmente, significativas para determinar cómo evolucionan las dinámicas de las respuestas locales y cómo los diferentes actores aprenden, reaccionan y se comportan estratégicamente en relación unos con otros, tanto a corto como a mediano y largo plazo.

Los actores siempre tienen, hasta cierto punto, expectativas sobre lo que es probable que suceda. Para el "público local", estas expectativas pueden adoptar diversas formas:

- En primer lugar, y de forma centralizada, las expectativas sobre la forma y el impacto del desarrollo de una propuesta de TER: cómo sería el desarrollo de un parque eólico o una planta de biomasa si se construyera en un territorio particular. Qué impactos locales buenos y malos podría tener, cómo podría funcionar en la generación de energía o en la mitigación del cambio climático, etc. Estas expectativas pueden estar más o menos desarrolladas en función de la situación de la tecnología, su familiaridad, su vigencia y su representación en los medios de comunicación.

- En segundo lugar, puede haber expectativas sobre los promotores del proyecto: cómo serán, cómo se comportarán y cómo deberían comportarse. Una vez más, estas expectativas podrían estar más o menos desarrolladas, y podrían estar potencialmente relacionadas con una empresa específica y un perfil local, regional o nacional que se conoce positiva o negativamente, o con una categoría más amplia de "industria", "gran empresa de servicios públicos", "gran multinacional", "propietario local" o "promotor local", con las asociaciones que acompañan a cada una de ellas.

- Tercero, pueden tener expectativas sobre el proceso que se va a seguir para examinar y luego tomar decisiones sobre una propuesta de desarrollo. Pueden tener expectativas normativas de lo que es justo, equitativo y correcto en la toma de decisiones (o formas de justicia procesal (Wolsink, 2007a); cómo deben comunicarse y participar; y también expectativas "realistas" sobre cómo será realmente el proceso, cómo podrían esperar ser excluidos o incluidos y cómo los flujos de información pueden o no ocurrir.

- En cuarto lugar, podrían tener expectativas acerca de lo que debería ser una distribución adecuada y apropiada de los beneficios de un desarrollo, y de lo que una comunidad. que potencialmente acoge un desarrollo, debería "obtener de él" (Wolsink, 2007b; Cass, Walker y Devine-Wright, 2010) en comparación con los otros actores.

Para los "protagonistas de la TER en las redes" también existen expectativas de diversas formas. Estas expectativas se comparten entre una diversidad de actores de la industria y de las políticas, y se han desarrollado de diversas maneras, incluyendo a través de la experiencia directa, la circulación de narrativas y las representaciones 
de los medios de comunicación. Entre estos actores se encuentra una expectativa generalizada de la posibilidad de hostilidad hacia la implementación de proyectos; a veces se materializa entre públicos particulares por razones particulares y en circunstancias y lugares particulares, y va acompañada de una orientación positiva del público en general hacia la idea de generar energía de manera renovable. Para algunos actores de las TER esto fue más allá, con la expectativa de que el público siempre se comportara como NIMBY en lugar de promover un impulso racional (Cass y Walker, 2009). Los actores de las TER también tienen expectativas sobre los procesos de desarrollo y de toma de decisiones, sobre cómo deben operar, cómo operan y cómo pueden y deben involucrarse con el público. Estas expectativas también se derivan de la experiencia en múltiples proyectos, y de escuchar de otros cómo se han llevado a cabo en diferentes localidades y con diferentes autoridades de planificación, donde los procesos pueden ser más comprensivos u hostiles a las aplicaciones de desarrollo.

Para los actores de las TER, y el público en general, es probable que estas expectativas sean anteriores a cualquier iniciación de una propuesta de desarrollo específica y pueden, en diferentes grados, estar arraigadas y resistirse al cambio. Sin embargo, se espera que estén, en parte, influenciados por las "interacciones" que surgen a medida que el público aprende acerca de las propuestas de proyectos, ve los informes de los medios de comunicación, asiste a las exposiciones, escucha acerca de los desarrolladores, etc. de modo que se puede esperar que sus expectativas evolucionen y cambien; a medida que los actores de las TER ven los informes de los medios de comunicación, se encuentran con la población local, y celebran exhibiciones y reuniones, sus expectativas acerca de los públicos y los procesos específicos de cada localidad pueden verse reforzadas, o pueden comenzar a cambiar o a modularse. Por lo tanto, las interacciones tienen consecuencias para las expectativas y viceversa.
En el lado derecho de la Figura 1, se considera que las características del lugar y de la comunidad determinan la forma en que los actores públicos desarrollan las expectativas y las estrategias de participación. Los sentidos del lugar, los apegos al lugar, y específicamente los apegos a los paisajes, han resultado ser importantes cuando se proponen las tecnologías de energía renovable (Devine-Wright y Howes, 2010). La naturaleza de las comunidades involucradas en términos de su sociodemografía, niveles de prosperidad, mezcla de residentes establecidos desde hace mucho tiempo y aquellos más recientes, y niveles de capital social también pueden ser significativos para explicar los patrones de expectativa y compromiso. Se destacan los contextos políticos, tanto locales y regionales como nacionales e internacionales, ya que pueden ser importantes para determinar los promotores y el apoyo financiero para el desarrollo de proyectos, para dar forma a los discursos, las estrategias de legitimación y compromiso que se emplean y para determinar los procesos y los límites de la toma de decisiones a través, por ejemplo, de la política de uso de la tierra (Ballesteros y Gallego, 2019).

Por último, se destaca el contexto empresarial y económico como determinante del compromiso, la tecnología y las estrategias de ubicación de los desarrolladores de energías renovables, sus relaciones con otros actores de las TER y su grado de sensibilidad a las respuestas del público. En la parte superior del modelo, el proceso formal de decisión para la aprobación de proyectos (que puede tomar varias formas dependiendo de la naturaleza, tamaño y ubicación del proyecto) se muestra como una aportación a los resultados de las decisiones. Es importante señalar aquí que las interacciones que se producen entre los actores de las TER y el público se muestran como una sola influencia que alimenta el proceso de toma de decisiones y los resultados. Distintos autores han señalado que los altos grados aparentes de actividad local, la anticipación de impactos negativos y la interacción multidimensional no necesariamente conducen a que los proyectos de TER sean rechazados y que, 
de manera similar, los patrones de desvinculación, la falta de interacción o la anticipación de resultados locales positivos no siempre conducen a la aprobación de proyectos (Bidwell, 2016a; Larson y Krannich, 2016; Bertsch et al., 2016; Ntanos et al., 2018). En general, se ha identificado que los factores que influyen en las decisiones de las autoridades locales, regionales o nacionales a cargo de la formulación y el desarrollo de proyectos energéticos no están directamente relacionados con el consentimiento del público local (Bidwell, 2016b; Bauwens y Devine-Wright, 2018).

\section{Conclusiones}

Después de una revisión extensa de la literatura, este trabajo resalta la necesidad de comprender la incidencia que tiene la ciudadanía en la formulación de las políticas públicas y en la construcción e implementación de las tecnologías de energías renovables TER. De modo que se hace necesario comprender desde una perspectiva compleja cómo los ciudadanos, denominados en este modelo actores, interactúan de manera imperante en la cadena de valor del mismo; en este sentido, es de suponerse que los nuevos desarrollos deben incluir no solo al público como eje de los modelos, sino que deben estar mediados por lo que este comprende y la manera en la que se relacionan científica y tecnológicamente, de tal forma que se forje un verdadero compromiso público que lleve a modificar las actitudes y comportamientos de las sociedades con respecto al uso y el abuso de la energía y sus respectivas fuentes. Desde este escenario, se presenta una propuesta que privilegia la necesidad de articular la acción pedagógica con la acción tecnológica para favorecer el desarrollo comunitario con la educación en energías renovables, entendida, en esencia, como el tratamiento de varios problemas y cuestiones relacionados con los recursos y las tecnologías de energía renovable como un área de conocimiento independiente (Kandpal y Broman, 2014), que aparece en el escenario educativo como una valiosa alternativa que debe edificarse para abordar los principales y más actuales problemas de educación en este campo, y sobre todo, en el contexto colombiano y latinoamericano. Lo que supone formular propuestas innovadoras en el campo de las relaciones ciencia-tecnología y sociedad, que implique a los ciudadanos como parte fundamental del modelo y sea parte de la simetría y no solo un contexto.

De esta forma, más allá de la presentación de resultados sobre investigaciones empíricas, existen otros intentos por describir y caracterizar las respuestas públicas a los proyectos de energías renovables y, también, elaborar marcos explicativos o modelos predictivos. Estos intentos incluyen las explicaciones del NIMBY de la oposición pública, que han gozado de una destacada presencia en reportajes de medios de comunicación prestantes y en debates políticos (Toynbee, 2007), los marcos teóricos para pensar en la aceptación social (Wüstenhagen et al., 2007), la tendencia cuantitativa de generar modelos estadísticos que caracterizan y miden las variables que predicen la naturaleza de la oposición pública (Wolsink, 2000) o la predicción de resultados de las decisiones (Toke, Breukers y Wolsink, 2008) hasta el más reciente modelo de Walker, que intenta representar la complejidad de procesos, dinámicas e interacciones que intervienen en la comprensión de las respuestas públicas a los desarrollos de proyectos TER a gran escala (Walker et al., 2014).

\section{Referencias}

Alsina, V., Martí, J. L. (2018). The birth of the crowd law movement: Tech-based citizen participation, legitimacy, and the quality of lawmaking. Analyse \& Kritik, 40(2), 337-358. https://doi.org/10.1515/ auk-2018-0019

Ballesteros-Ballesteros, V. A., Gallego-Torres, A. P. (2019). Model of education in renewable energies from the public engagement and the energetic attitude. Revista Facultad de Ingeniería, 28(52), 27-42. https://doi.org/10.19053/01211129.v28. n52.2019.9652 
Batel, S. (2020). Research on the social acceptance of renewable energy technologies: Past, present and future. Energy Research \& Social Science, 68. https://doi.org/10.1016/j.erss.2020.101544

Bauwens, T., Devine-Wright, P. (2018). Positive energies? An empirical study of community energy participation and attitudes to renewable energy. Energy Policy, 118, 612-625. https://doi.org/10.1016/j. enpol.2018.03.062

Berg, M., Lidskog, R. (2018). Deliberative democracy meets democratized science: A deliberative systems approach to global environmental governance. Environmental Politics, 27(1), 1-20. https://doi. org/10.1080/09644016.2017.1371919

Bertsch, V., Hall, M., Weinhardt, C., Fichtner, W. (2016). Public acceptance and preferences related to renewable energy and grid expansion policy: Empirical insights for Germany. Energy, 114, 465-477. https://doi.org/10.1016/j.energy.2016.08.022

Bidwell, D. (2016a). The effects of information on public attitudes toward renewable energy. Environment and Behavior, 48(6), 743-768. https://doi. org/10.1177/0013916514554696

Bidwell, D. (2016b). Thinking through participation in renewable energy decisions. Nature Energy, 1, e16051. https://doi.org/10.1038/nenergy.2016.51

Brown, M. T., Ulgiati, S. (1999). Emerge evaluation of the biosphere and natural capital. AMBIO A Journal of the Human Environment, 28(6), 1-15

Burningham, K., Barnett, J., Walker, G. (2015). An array of deficits: Unpacking NIMBY discourses in wind energy developers' conceptualizations of their local opponents. Society \& Natural Resources, 28(3), 246-260. https://doi.org/10.1080/08941920.2014. $\underline{933923}$

Cardullo, P., Kitchin, R. (2018). Being a "citizen" in the smart city: up and down the scaffold of smart citizen participation in Dublin, Ireland. Geo/ournal, $84,1-13$

Cass, N., Walker, G. (2009). Emotion and rationality: The characterization and evaluation of opposition to renewable energy projects. Emotion, Space and Society, 2(1), 62-69. https://doi.org/10.1016/j. emospa.2009.05.006
Cass, N., Walker, G., Devine-Wright, P. (2010). Good neighbors, public relations, and bribes: the politics and perceptions of community benefit provision in renewable energy development in the UK. Journal of Environmental Policy \& Planning, 12(3), 255-275. https://doi. org/10.1080/1523908X.2010.509558

Devine-Wright, P., Howes, Y. (2010). Disruption to place attachment and the protection of restorative environments: A wind energy case study. Journal of Environmental Psychology, 30(3), 271-280. https:// doi.org/10.1016/j.jenvp.2010.01.008

Dinica, V. (2018). Public engagement in governance for sustainability: A two-tier assessment approach and illustrations from New Zealand. Public Management Review, 20(1), 23-54. https://doi.org/10.108 0/14719037.2017.1293142

Firestone, J., Kempton, W. (2007). Public opinion about large offshore wind power: Underlying factors. Energy Policy, 35(3), 1584-1598. https://doi.org/10.1016/j.enpol.2006.04.010

Franco Moreno, R. A., Reina Hernández, J. A., Riveros Toro, C. M. (2020). Concepciones sobre química verde en profesores de química en formación inicial. Noria: Investigación Educativa, 1(5), 94-108

Furlan, C., Mortarino, C. (2018). Forecasting the impact of renewable energies in competition with non-renewable sources. Renewable and Sustainable Energy Reviews, 81, 1879-1886. https://doi.org/10.1016/j.rser.2017.05.284

Futrell, R. (2003). Framing processes, cognitive liberation, and NIMBY protest in the US chemical-weapons disposal conflict. Sociological Inquiry, 73(3), 359386. https://doi.org/10.1111/1475-682X.00061

Gustafsson, S., Ivner, J., Palm, J. (2015). Management and stakeholder participation in local strategic energy planning: Examples from Sweden. Journal of Cleaner Production, 98, 205-212. https://doi.org/10.1016/j.jclepro.2014.08.014

Hsieh, T., Vaickus, M. H., Remick, D. G. (2018). Enhancing scientific foundations to ensure reproducibility: A new paradigm. The American Journal of Pathology, 188(1), 6-10. https://doi.org/10.1016/j. ajpath.2017.08.028 
James, J. (2019). Strength benefit of sawdust/wood ash amendment in cement stabilization of an expansive soil. Revista Facultad de Ingeniería, 28(50), 44-61. https://doi.org/10.19053/01211129.v28. $\underline{\mathrm{n} 50.2019 .8790}$

Kandpal, T. C., Broman, L. (2014) Renewable Energy Education: A Global Status Review. Renewable and Sustainable Energy Reviews, 34, 300-324. https:// doi.org/10.1016/j.rser.2014.02.039

Kreimer, P., Vessuri, H. (2018). Latin American science, technology, and society: A historical and reflexive approach. Tapuya: Latin American Science, Technology and Society, 1(1), 17-37. https://doi.org/10.10 80/25729861.2017.1368622

Kropp, C. (2018). Controversies around energy landscapes in third modernity. Landscape Research, 43(4), 562-573. https://doi.org/10.1080/01426397.2017. 1287890

Larson, E., Krannich, R. (2016). "A great idea, just not near me!" Understanding public attitudes about renewable energy facilities. Society \& Natural Resources, 29(12), 1436-1451. https://doi.org/10.108 0/08941920.2016.1150536

Li, G., Zheng, X. (2016). Thermal energy storage system integration forms for a sustainable future. Renewable and Sustainable Energy Reviews, 62, 736-757. https://doi.org/10.1016/j.rser.2016.04.076

Lilliestam, J., Hanger, S. (2016). Shades of green: Centralization, decentralization, and controversy among European renewable electricity visions. Energy Research \& Social Science, 17, 20-29. https://doi.org/10.1016/j.erss.2016.03.011

Lyall, C., Tait, J. (2017). New Modes of Governance: Developing an Integrated Policy Approach to Science, Technology, Risk, and the Environment. Routledge.

MacFarlane, J. M. (2018). Managing the future imaginary: Does 'post-normal' science need public relations? RT: A Journal on Research Policy and Evaluation, 6(1), 1-9. https://doi. org/10.13130/2282-5398/9139

Martin, V. Y. (2017). Citizen science as a means for increasing public engagement in science: Presumption or possibility? Science Communication, 39(2), 142168. https://doi.org/10.1177/1075547017696165
Mor, N., Reich, Z. (2018). From "Trust Me" to "Show $M^{\prime \prime}$ Journalism: Can DocumentCloud help to restore the deteriorating credibility of news? Journalism Practice, 12(9), 1091-1108. https://doi.org/10. 1080/17512786.2017.1376593

Neshkova, M. I., Guo, H. (2018). Policy target populations and public participation in agency decision making. International Public Management Journal, 21(2), 297-325. https://doi.org/10.1080/10967494. 2016.1160012

Ntanos, S., Kyriakopoulos, G., Chalikias, M., Arabatzis, G., Skordoulis, M. (2018). Public perceptions and willingness to pay for renewable energy: A case study from Greece. Sustainability, 10(3), e687. https://doi.org/10.3390/su10030687

Ortega-Quevedo, V., Gil Puente, C. (2019). La naturaleza de la ciencia y la tecnología: una experiencia para desarrollar el pensamiento crítico. Científica, 35(2), 167-182. https://doi. org/10.14483/23448350.14095

Petrova, M. A. (2016). From NIMBY to acceptance: Toward a novel framework VESPA for organizing and interpreting community concerns. Renewable Energy, 86, 1280-1294. https://doi.org/10.1016/j. renene.2015.09.047

Rees, W., Wackernagel, M. (1996). Urban ecological footprints: Why cities cannot be sustainable and why they are a key to sustainability. Environmental Impact Assessment Review, 16(4-6), 223-248. https://doi.org/10.1016/S0195-9255(96)00022-4

Rip, A. (2018). Science institutions and grand challenges of society: A scenario. In Futures of Science and Technology in Society (pp. 35-47). Springer

Robayo-Salazar, R. A., Mejía de Gutiérrez, R., Mulford-Carvajal, A. J. (2016). Production of building elements based on alkali-activated red clay brick waste. Revista Facultad de Ingeniería, 25(43), 21-30. https:// doi.org/10.19053/01211129.v25.n43.2016.5294

Salamanca-Céspedes, J. E. (2019). La educación en energías renovables no convencionales en la formación de ingenieros electrónicos. Noria: Investigación Educativa, 2(4), 11-18.

Schot, J., Rip, A. (1997). The past and future of constructive technology assessment. Technological 
Forecasting and Social Change, 54(2-3), 251-268. https://doi.org/10.1016/S0040-1625(96)00180-1

Speer, P. W., Han, H. (2018). Re-engaging social relationships and collective dimensions of organizing to revive democratic practice. Journal of Social and Political Psychology, 6(2), 745-758. https://doi. org/10.5964/jspp.v6i2.929

Toke, D., Breukers, S., Wolsink, M. (2008). Wind power deployment outcomes: How can we account for the differences? Renewable and Sustainable Energy Reviews, 12(4), 1129-1147. https://doi.org/10.1016/j. rser.2006.10.021

Toynbee, P. (2007). Nimbys can't be allowed to put a block on wind farms. The Guardian. http://www. theguardian.com/commentisfree/2007/jan/05/ comment.politics

Walker, G. (1995). Renewable energy and the public. Land Use Policy, 12(1), 49-59. https://doi. org/10.1016/0264-8377(95)90074-C

Walker, G., Cass, N. (2007). Carbon reduction, "the public" and renewable energy: engaging with socio-technical configurations. Area, 39(4), 458-469. https://doi.org/10.1111/j.1475-4762.2007.00772.x

Walker, G., Cass, N., Burningham, K., Barnett, J. (2010). Renewable energy and sociotechnical change: imagined subjectivities of 'the public' and their implications. Environment and Planning A: Economy and Space, 42(4), 931-947. https://doi.org/10.1068/a41400

Walker, G., Devine-Wright, P., Barnett, J., Burningham, K., Cass, N., Devine-Wright, H., Speller, G., Barton, J. P., Evans, B., Heath, Y., Infield, D., Parks, J., Theobald, K. (2014). Symmetries, expectations, dynamics and contexts: A framework for understanding public engagement with renewable energy projects. In P. Devine-Wright (Ed.), Renewable Energy and the Public: From NIMBY to Participation (pp.33-46). Routledge.

Wilsdon, J., Willis, R. (2004). See-through Science: Why Public Engagement Needs to Move Upstream (1st ed.). Demos (London). https://doi.org/10.13140/ RG.2.1.3844.3681

Wilsdon, J., Wynne, B., Stilgoe, J. (2005). The Public Value of Science (or How to Ensure that Science ReaIly Matters) (1st ed.). Demos (London). https://doi. org/10.13140/RG.2.1.2281.7449

Wolsink, M. (2000). Wind power and the NIMBY-myth: Institutional capacity and the limited significance of public support. Renewable Energy, 21(1), 49-64. https://doi.org/10.1016/S0960-1481(99)00130-5

Wolsink, M. (2007a). Planning of renewables schemes: Deliberative and fair decision-making on landscape issues instead of reproachful accusations of non-cooperation. Energy Policy, 35(5), 2692-2704. https://doi.org/10.1016/j.enpol.2006.12.002

Wolsink, M. (2007b). Wind power implementation: The nature of public attitudes: equity and fairness instead of 'backyard motives'. Renewable and Sustainable Energy Reviews, 11(6), 1188-1207. https:// doi.org/10.1016/j.rser.2005.10.005

Wüstenhagen, R., Wolsink, M., Bürer, M. J. (2007). Social acceptance of renewable energy innovation: An introduction to the concept. Energy Policy, 35(5), 2683-2691. https://doi.org/10.1016/j. enpol.2006.12.001 